\title{
Isochronous explicit time integration framework: illustration to thermal stress problems involving both first- and second-order transient systems
}

\begin{abstract}
In this article, an isochronous explicit time integration framework (or the explicit ilntegrator) for solving thermal stress problems is illustrated. Similar to the implicit case, the same adaptation process of the isochronous integration is valid for the explicit case. That is, the adaptation process endows the explicit version of the generalized single step family of algorithms for second-order systems (explicit GS4-2 family of algorithms) with the applicability to first-order systems, and the explicit version of the GS4 family of algorithms for first-order systems (explicit GS4-1 family of algorithms) is automatically generated. Two illustrative thermal stress dynamic applications are shown to demonstrate the practicality and convenience of the explicit integrator.
\end{abstract}

Keyword: Dynamics; Explicit algorithms; First order systems; Second order systems; Thermal stress waves; Time integration 